\title{
Bounds on neutron-mirror neutron mixing from pulsar timing
}

\author{
I. Goldman, ${ }^{2,1}$ R. N. Mohapatra, ${ }^{3}$ and S. Nussinov ${ }^{1}$ \\ ${ }^{1}$ School of Physics and Astronomy, Tel Aviv University, 6997801 Tel Aviv, Israel \\ ${ }^{2}$ Department of Physics, Afeka Tel Aviv Engineering College, 6997801 Tel Aviv, Israel \\ ${ }^{3}$ Maryland Center for Fundamental Physics and Department of Physics, University of Maryland, \\ College Park, 20742 Maryland, USA
}

(Received 5 February 2019; published 27 December 2019)

\begin{abstract}
The possibility of neutron-mirror neutron transition has recently been discussed both from theoretical and experimental perspectives. We point out that such a transition would imply mass loss in neutron stars leading to a significant change of the orbital period of neutron star binary systems. Known limits on the period changes of such binaries therefore put strong limits on the above transition rate and hence on the $n-n^{\prime}$ mixing parameter $\epsilon^{\prime}$. These considerations exclude a large range of $\epsilon^{\prime}$ even if the $n-n^{\prime}$ mass difference is $\delta m^{\prime}$ as large as a few MeV. Our limits also exclude a large range of the values required to explain the neutron decay anomaly via $n-n^{\prime}$ mixing.
\end{abstract}

DOI: 10.1103/PhysRevD.100.123021

\section{INTRODUCTION}

The possibility that there may be a mirror sector of the standard model with identical particle content and gauge symmetry as the standard model [1] has received a great deal of attention. In particular, the dark matter of the Universe as well as any sterile neutrinos may be the lightest baryon (or atom) and the light neutrinos of the mirror sector, respectively. Prior to symmetry breaking, these models assume the existence of $Z_{2}$ (mirror) symmetry between the two sectors, which keeps the same number of parameters even though the number of particles is doubled, making this a very economical scenario of beyond the standard model physics. Two realizations of these theories have been extensively discussed: one where mirror symmetry is respected by the electroweak vacuum expectation values (VEVs) [2] and another where the mirror symmetry is broken via different electroweak scales in the two sectors [3]. In this paper, we focus on the first class of models where $v_{w k}=v_{w k}^{\prime}$ (where $v_{w k}$ and $v_{w k}^{\prime}$ refer to the standard model and the mirror SM Higgs VEVs); we use a prime to denote the entities in the mirror sector). In this case, we have identical microphysics and the same fermion masses in both sectors. There is, however, a generic problem with both of these scenarios in that the extra neutrinos $\left(\nu^{\prime}\right)$ and photons $\left(\gamma^{\prime}\right)$ from the mirror sector contribute too much to

Published by the American Physical Society under the terms of the Creative Commons Attribution 4.0 International license. Further distribution of this work must maintain attribution to the author(s) and the published article's title, journal citation, and DOI. Funded by SCOAP ${ }^{3}$. the number of degrees of freedom at the big bang nucleosynthesis (BBN) epoch, destroying the success of the $\mathrm{BBN}$ predictions. A cure for this is to assume breaking of the $Z_{2}$ symmetry in the early Universe so as to have asymmetric inflationary reheating in the two sectors, resulting in a lower reheat temperature $\left(T^{\prime}\right)$ of the mirror sector compared to that $(T)$ of the visible one [4]. This breaking eventually trickles down to the low energies, leading, in general, to a splitting of the mirror and visible fermion masses [5]. The above-mentioned symmetric picture could, however, remain almost exact if the asymmetric inflation picture is carefully chosen. The cosmology of such scenarios has been discussed in $[6,7]$.

An interesting new phenomenon is possible in almost exact mirror models, if there are interactions mixing the neutron with the mirror neutron state (denoted by $\epsilon_{n-n^{\prime}} \equiv \epsilon^{\prime}$ ). In such a case, one can expect $n \rightarrow n^{\prime}$ oscillations to take place in the laboratory [8], and indeed there are ongoing and already completed searches for such oscillations $[9,10]$ at various neutron facilities.

We note that $n-n^{\prime}$ oscillation is similar to the neutronantineutron oscillation suggested very early [11] and extensively discussed in the literature [12]. For $n-\bar{n}$ oscillation to occur, one needs a $\Delta B=2$ interaction that leads to a mixing mass $\epsilon_{n-\bar{n}}$ between the $n$ and $\bar{n}$ similar to $\epsilon^{\prime}$. There is, however, a major difference between these two oscillations. The mass equality of the neutron and antineutron is guaranteed by the $C P T$ theorem, known to be an exact consequence of local Lorentz invariant quantum field theories. However, the mass degeneracy between $n$ and $n^{\prime}$ oscillations requires an almost exact $Z_{2}$ symmetry, which as discussed above, needs to be a weakly broken symmetry to satisfy big bang nucleosynthesis constraints. 
With stringent upper bounds on $\epsilon_{n \bar{n}}$, the neutron-antineutron mixings have been obtained by searching for $n \bar{n}$ conversion in magnetically shielded neutron beams [13] and in nuclei [14]. The subsequent annihilation of the generated $\bar{n}$ can be readily identified in both cases. The $(n \rightarrow \bar{n})$ transitions in nuclei are highly suppressed by the ratio $\epsilon_{n \bar{n}}^{2} /(E-\bar{E})^{2}$, with $\epsilon_{n \bar{n}}=1 / \tau_{n \bar{n}}$ being the offdiagonal $\Delta B=2$ element of the $2 \times 2$ energy-mass matrix for the $n-\bar{n}$ system and $E-\bar{E}$ the large difference between the diagonal elements-the energies of the neutron and antineutron in the nucleus. Still, the large number of neutrons in the underground detectors compensates this enormous suppression, leading quite remarkably to the same upper limit as is obtained from oscillation searches:

$$
\epsilon_{n \bar{n}}=1 / \tau_{n \bar{n}}<10^{-8} \mathrm{sec}^{-1} \quad \text { or } \quad 10^{-23} \mathrm{eV}
$$

For the case of $\epsilon^{\prime}$, however, the analogous bounds are far weaker. The $n^{\prime}$ generated in neutron beams or bottles simply leave the system, manifesting only in a deficiency of neutrons beyond what is expected from the neutron decay [9]. The analysis in this case is further complicated by the possible presence of mirror magnetic fields which can suppress the transition rate and, unlike ordinary B fields, cannot be shielded. Also unlike for $n \rightarrow \bar{n}, n \rightarrow n^{\prime}$ transitions inside nuclei are energetically forbidden as the neutrons are bound (typically by $\sim 8 \mathrm{MeV}$ ) and the equal mass mirror neutrons with no (ordinary) nuclear interactions are unbound.

In this paper, we focus on the possibility that $n \rightarrow n^{\prime}$ transitions can lead to mass loss of a neutron star, and if the latter star is a member of the binary star system, then this mass loss affects the binary period. Indeed [15] the mass loss of any kind of such neutron stars implies an increase of the orbital period of the binary system $P_{b}$. The currently available limits on $\left|\dot{P}_{b} / P_{b}\right|$ of binary neutron star systems yield stringent limits on the $n-n^{\prime}$ mixing parameter $\epsilon^{\prime}$ for a large range of $n-n^{\prime}$ mass difference, $\delta m$.

The original suggestion [16] was motivated by the hope that $\left(n \rightarrow n^{\prime}\right)$ oscillations generating completely mixed $n n^{\prime}$ stars may explain some peculiarities of the observed mass distribution of neutron stars. To this end two conditions have to be satisfied:

(a) The mass reduction in the transition from a pure neutron star to a completely mixed $n n^{\prime}$ should be substantial $(\sim 30 \%)$.

(b) The timescale for this transition should be shorter than the ages of the observed neutron stars.

Condition (a) involves only standard model physics, specifically the nuclear equations of state, and is difficult to satisfy. Condition (b) depends on the new physics parameter $\epsilon^{\prime}$. Our new bounds on $\epsilon^{\prime}$ tend to exclude it.

Our limits on $\epsilon^{\prime}$ also exclude the possibility that $n n^{\prime}$ oscillations can explain the neutron decay anomaly [17].

\section{TRANSITION OF NEUTRON STAR TO MIXED NEUTRON-MIRROR NEUTRON STARS INDUCED BY $n-n^{\prime}$ MIXINGS}

We start by discussing some broad issues with the $n-n^{\prime}$ transition in a neutron star and its implications. A key point is that $n \rightarrow n^{\prime}$ transitions, kinematically forbidden in nuclei, do occur in neutron stars. The reason for this is that the neutrons in neutron stars are mainly bound by gravity and $n o t$ by nuclear forces. Let us suppose that an $n \rightarrow n^{\prime}$ conversion occurred at some point in the star. Under the pressure which is proportional to the energy density, a neighboring neutron rushes into the "hole" generated by the converted neutron, gaining, in the process, kinetic energy which is of order of the Fermi energy $E_{F}$. Further energy is gained when the produced $n^{\prime}$ gravitates to the center of the star and a surface neutron replaces the neutron which went into the above hole. Therefore, the net (eventually radiated) energy stemming from an early, single $n n^{\prime}$ transition is

$$
m_{n}\left(e^{\phi(R)}-e^{\phi(0)}\right)+\left\langle E_{F}\right\rangle
$$

where $e^{\phi(r)}=g_{o o}^{1 / 2}(r), e^{\phi(R)}=\left(1-2 G M / c^{2}\right)^{1 / 2}$, and $\left\langle E_{F}\right\rangle$ is the average Fermi energy of the neutron that disappeared and $M$ and $R$ are the mass and the radius of the original neutron star.

In reality we have continuous inward drifting of both mirror neutrons $\left(n^{\prime}\right)$ and neutrons $(n)$ due to the ongoing $n \rightarrow n^{\prime}$ transitions over the whole volume of the star. This also causes the star to shrink, which significantly increases the gravitational binding.

To estimate the resulting mass decrease, we numerically solve the Tollman-Oppenheimer-Volkov equations with a commonly used equation of state [18]. Starting with neutron stars of masses $M 1.57-1.94 M_{\odot}$, we found (as detailed in Sec. IV) a 9\%-14\% decrease after the transition to a completely symmetric mixed star.

The rate of transition to a mixed star is estimated in two steps. These correspond to the two factors comprising the $n n^{\prime}$ transition rate

$$
\Gamma\left(n \rightarrow n^{\prime}\right)=\Gamma(n n) P_{n n^{\prime}}
$$

where $\Gamma_{n n}$ is the rate of $n n$ collisions and $P_{n n^{\prime}}$ is the probability of having $n^{\prime}$ (rather than the original neutron $n$ ) at the time of the collision.

Equation (2) reflects a simple, physically motivated picture [19] in which one assumes the following:

(a) The coherent buildup of the $\left|n^{\prime}\right\rangle$ component in the initial purely $|n\rangle$ state of the two-component system proceeds unimpeded by nuclear interactions during the time of flight between two consecutive collisions.

(b) The coherent buildup stops upon collision, and the $n^{\prime}$ part is released as outgoing mirror neutron particles. The free evolution of the initial pure neutron state during the short time of flight starting with 
$|\psi(0\rangle=| n\rangle=\left(\begin{array}{l}1 \\ 0\end{array}\right)$ yields $|\psi(t)\rangle=e^{(i H t)}|\psi(0)\rangle \approx(1+$ $i H t)|\psi(0)\rangle \approx\left(\begin{array}{c}1 \\ i \epsilon^{\prime} t\end{array}\right)$ where $H=\Delta(E) \sigma_{z}+\epsilon^{\prime} \sigma_{x}$ is the Hamiltonian in the two-dimensional $|n\rangle,\left|n^{\prime}\right\rangle$ Hilbert space. This then yields a probability of generating a mirror neutron $n^{\prime}$ between two consecutive collisions of $P_{n n^{\prime}}=\left[\epsilon^{\prime} \cdot t_{n n}\right]^{2}$, an expression valid for times $t_{n n}$ shorter than $\left(\epsilon^{\prime}\right)^{-1}$ and for $(\Delta E) t_{n n} \leq 1$. As is well known from the case of $n-\bar{n}$ oscillation, the dependence on $\Delta E$, the energy difference between the two states, cancels out in the limit of short times $t_{n n}$.

Substituting the above $P_{n n^{\prime}}$ and $\Gamma_{n n}=t_{n n}^{-1}$ in Eq. (2), we find

$$
\Gamma_{n \rightarrow n^{\prime}}=t_{n n} \epsilon^{\prime 2}
$$

Using $t_{n n} \approx 10^{-23} \mathrm{sec}$, the time required to travel the $O$ (Fermi) distance between neighboring neutrons at $\sim 1 / 3$ the speed of light then leads to an estimate of the conversion rate

$$
\Gamma_{n \rightarrow n^{\prime}}=0.6 \times 10^{-7}\left[\epsilon^{\prime} / 10^{-11} \mathrm{eV}\right]^{2} \mathrm{yr}^{-1} .
$$

Clearly, this is a very rough estimate. In particular, having the nuclear medium manifest just as a series of frequent collisions is very crude. The conversion is ongoing, and the proper treatment should use the Schrödinger equation in the medium as in the careful treatment of $n \rightarrow \bar{n}$ nuclear transitions in [20]. That careful treatment yielded $n \bar{n}$ transitions in nuclei which are somewhat faster than the estimates of [19] followed here. This suggests that indeed our Eq. (4) does not overestimate the rate of $n n^{\prime}$ transitions in the neutron star as well.

The Pauli blocking effects (which may be quite important) increase $t_{n n}$. Neglecting it as we did above then yields a more conservative estimate.

The TOV equations and the corresponding equilibrium states keep changing in time as more and more neutrons are being converted to mirror neutrons. These mirror neutrons keep drifting towards the center, generating an equally mixed region of a lower energy. The equally mixed spherical region gradually expands and eventually overtakes the whole star. Here, we will not delve into all of these details.

\section{ASTROPHYSICAL CONSTRAINTS ON THE NEUTRON STAR TO MIXED STAR TRANSITIONS}

We now describe our main result. It involves utilizing the precise measurement of the change of the orbital period pulsars in binary systems to limit $\epsilon^{\prime}$. Such a period increase, which is expected whenever the mass of either of the binary members decreases, was first noted in 1925 by Jeans [21]. He obtained a simple expression for the rate of change of binary orbital period $P_{b}$ as a function of the mass change:

$$
\frac{d P_{b} / d t}{P_{b}}=-2 \frac{d M / d t}{M}
$$

where $M=M_{1}+M_{2}$.

While Jeans envisioned mass loss due to the electromagnetic radiation emitted by the stars, the above relation holds for any form of "radiation" e.g., via neutrinos, axions, mirror photons, and other forms of mass loss—-so long as the emission from either member of the binary is symmetric in its rest frame.

(As noted in Ref. [15], if some fraction of the energy is emitted electromagnetically-most likely as x rays-even more stringent limits would be obtained.)

\section{A. Limits from change of binary period}

We next present the limits on mass loss for the following binary pulsars.

PSR $1916+13$.-We use the data of Weisberg et al. (2010). The spin-down age of the pulsar is $1.1 \times 10^{8} \mathrm{yr}$. It is commonly assumed that the pulsar companion is a neutron star, so there is no mass transfer between the binary members. Also, one neglects the mass accretion from the interstellar medium. After accounting for the expected gravitational radiation, galactic acceleration, and further dynamical corrections, there is still some limited room for a positive change of binary period that could have followed from the mass decrease:

$$
\frac{\dot{P}_{b}}{P_{b}}<1.36 \times 10^{-11} \mathrm{yr}^{-1}
$$

implying

$$
\frac{\dot{M}}{M}<6.8 \times 10^{-12} \mathrm{yr}^{-1}
$$

for the two neutron stars losing mass.

PSR J1141-6545.- This is a young pulsar of about $2 \times 10^{6} \mathrm{yr}$. The companion is a massive white dwarf of mass $0.98 M_{\odot}$, and the neutron star has a mass $1.3 M_{\odot}[22,23]$.

This system is a superb laboratory for testing GR. Using the data from the above observational papers, one finds that the residual (subtracting from the measured value the gravitational radiation terms as well as the galactic acceleration and the kinematic effect and allowing for the uncertainties of all of the above) positive possible value of the orbital period rate of change is very small,

$$
\frac{\dot{P}_{b}}{P_{b}}<1.84 \times 10^{-12} \mathrm{yr}^{-1},
$$

implying for the given masses and taking into account that only the neutron star is undergoing the mass loss 


$$
\frac{\dot{M}}{M}=\frac{M+M_{c}}{2 M} \frac{\dot{P}_{b}}{P_{b}}<1.6 \times 10^{-12} \mathrm{yr}^{-1}
$$

where $M_{c}$ is the mass of the white dwarf companion. This result is very important, as for the previously mentioned pulsars, one may have argued that the transition $n \rightarrow n^{\prime}$ has already finished. This young pulsar closes the door on this argument.

PSR J0437 - 4715.-This is a neutron star-white dwarf binary with masses of $1.76 M_{\odot}$ and $0.25 M_{\odot}$, respectively. The spin-down age is $1.6 \times 10^{9} \mathrm{yr}$. Using the data from the above observational papers, one finds that the residual (subtracting from the measured value the gravitational radiation terms as well as the galactic acceleration and the kinematic effect and allowing for the uncertainties of all of the above) positive possible value of the orbital period rate of change is [23]

$$
\frac{\dot{P}_{b}}{P_{b}}<2.8 \times 10^{-11} \mathrm{yr}^{-1}
$$

implying

$$
\frac{\dot{M}}{M}<1.6 \times 10^{-11} \mathrm{yr}^{-1}
$$

PSR J1952 + 2630.-This pulsar is in a binary orbit with a (0.93-1.4) $M_{\odot}$ white dwarf companion [24]. Its spin-down age is $7.7 \times 10^{7} \mathrm{yr}$, the orbital period is 0.39 days, and during 800 days of follow-up, the error on the period is $7 \times 10^{-12}$ days. This leads to $\frac{\dot{P}_{b}}{P_{b}}<8.2 \times 10^{-12} \mathrm{yr}^{-1}$.

Taking into account that only the pulsar loses mass, we get

$$
\frac{\dot{M}}{M}=\left(\frac{M+M_{c}}{2 M}\right) \frac{\dot{P}_{b}}{P_{b}}<7 \times 10^{-12} \mathrm{yr}^{-1}
$$

where $M_{c}$ is the mass of the white dwarf companion. The expected period change due to gravitational radiation in this pulsar is 2 orders of magnitude smaller and thus does not interfere with the derived limit.

We next briefly discuss limits that follow from the observations of old neutron stars which tend to exclude the possibility that they have completely converted to mixed neutron-mirror neutron stars.

\section{B. Massive millisecond pulsars}

There is a class of old, (1-10) Gyr, millisecond binary pulsars with masses in the range (2-2.5) $M_{\odot}$ (see [25] and references therein). In what follows we present one such example.

PSR B $1957+20$. - This is a millisecond pulsar with a mass of $\sim 2.4 M_{\odot}$, a companion white dwarf with mass $0.025 M_{\odot}$ and spin-down age of $1.6 \times 10^{9} \mathrm{yr}$ [26]. If the conversion occurred during this time, then we find, using the results of the next section, that it must have started with an extremely large mass of $\sim 3 M_{\odot}$, a value well above the maximal mass allowed by realistic equations of state.

This pulsar (and also the binary neutron star merger and ensuing gravity waves) yields limits on $\epsilon^{\prime}$ which are weaker than those detailed above. They are, however, of some interest here and, in particular, further exclude the scenario of [16].

\section{Gravitational waves observations}

The recent observation (in gravitational waves and in much of the electromagnetic spectrum) of a neutron star binary merger may also be relevant to our discussion. In the scenario of Ref. [16], such mergers are likely to involve stars which are already mixed.

According to Sec. IV below, the radius of the mixed star is reduced by a factor $f \sim 0.7$ relative to the original radius of the initial neutron star. For approximately circular orbits, the rate of energy loss via gravitational radiation is

$$
d W / d t=\frac{32 G_{N}}{5 c^{5}} \mu^{2} \Omega_{\mathrm{orb}}^{6} a^{4}
$$

where $\mu$ is the reduced mass: $\mu=\left(M_{1} M_{2}\right) /\left(M_{1}+M_{2}\right)$. Shrinking the radii of both stars $R_{1}$ and $R_{2}$ by the factor $f$ will decrease the orbit size at the time of merging $a=$ $R_{1}+R_{2}$ by the same factor $f$. Using Kepler's law $\Omega_{\text {orb }} \sim a^{-3 / 2}$, we find that $d W / d t$ will increase by a factor of $f^{-5}$. We also have $\sim 12 \%$ of $\mu$, which will tend to reduce this increase. We find a net enhancement of the instantaneous $\frac{d W}{d t}$ of about four. Thus, the observed pattern of luminosity and frequency may then conflict with the fitted gravitational waves templates.

\section{CALCULATIONS OF MASS AND RADIUS REDUCTION DUE TO THE TRANSITION TO A FULLY MIXED STAR}

To calculate the fractional mass and radius reductions resulting from the transition to a completely mixed star, we employ three initial neutron star configurations and solve the general relativistic TOV structure equations. The $n n^{\prime}$ transition conserves the sum of the neutron number $N$ and the number of mirror neutrons $N^{\prime}$. Thus, each of these cases corresponds to a specific total baryonic mass $M_{b}=$ $\left(N+N^{\prime}\right) m_{n}$. In the TOV equations we use the highly cited realistic nuclear equation of state of [18].

For the original pure neutron star, we use the standard TOV equations, whereas for the completely mixed star, we use the TOV equations for two fluids which interact only gravitationally as in $[27,28]$. The results are as follows: 


$$
M_{b}=1.81 M_{\odot}:
$$

$$
\begin{aligned}
M & =1.57 M_{\odot}, \quad R=12.2 \mathrm{~km}, \quad e^{\phi(R)}-e^{\Phi(0)}=0.15, \\
M_{n n^{\prime}} & =1.43 M_{\odot}=0.91, \quad R_{n n^{\prime}}=8.8 \mathrm{~km}=0.72 R .
\end{aligned}
$$

$M_{b}=2.17 M_{\odot}:$

$$
\begin{aligned}
M & =1.82 M_{\odot}, \quad R=12.37 \mathrm{~km}, \quad e^{\phi(R)}-e^{\Phi(0)}=0.17, \\
M_{n n^{\prime}} & =1.62 M_{\odot}=0.89 M, \quad R_{n n^{\prime}}=8.8 \mathrm{~km}=0.71 R .
\end{aligned}
$$

$M_{b}=2.44 M_{\odot}:$

$$
\begin{aligned}
M & =1.97 M_{\odot}, \quad R=12.4, \quad e^{\phi(R)}-e^{\Phi(0)}=0.19 \\
M_{n n^{\prime}} & =1.7 M_{\odot}=0.86 M, \quad R_{n n^{\prime}}=8.65 \mathrm{~km}=0.7 R .
\end{aligned}
$$

Here, the first line in each case presents the mass $M$ and radius $R$ of the pure neutron star with baryonic mass $M_{b}$, $e^{\phi(r)}=g_{00}(r)^{1 / 2}$. The second line represents the mass and radius, $M_{n n^{\prime}}$ and $R_{n n^{\prime}}$, of the corresponding completely mixed star.

We find that typical final masses are $0.91-0.86$ of the corresponding initial mass, which seems insufficient for the scenario in [16].

\section{BOUNDS ON $\epsilon^{\prime}$ FROM PULSAR PERIOD INCREASE MEASUREMENTS}

Returning to the main goal of the paper, namely, limiting $\epsilon^{\prime}$, we need to relate $\frac{1}{N} \frac{d N}{d t}=\Gamma_{n n^{\prime}} \propto \epsilon^{\prime 2}$, the rate of neutron to mirror neutron transition to the stellar $\frac{\dot{M}}{M}$. Since neutrons from the entire volume of the neutron star can transform to $n^{\prime}$, the resulting total mass loss of the neutron star due to this is proportional to $\Gamma_{n \rightarrow n^{\prime}}$.

In general, only neutron stars which are still in the process of transiting to a mixed star will exhibit mass loss. A priori, for extremely "high" $\Gamma_{n n^{\prime}}$, some of the older observed pulsars in binaries may be "too old."

A great advantage is that all the different binary pulsars (with a variety of masses, spin-down ages, and companions) should conform to a single fundamental parameter $\epsilon^{\prime}$. Thus, we can use the youngest pulsars to set stringent limits on $\epsilon^{\prime}$. In turn, this implies that the older pulsars are also still in the process of transition. In particular, for small enough values of $\epsilon^{\prime}$, even these older pulsars may be at the very initial stages of the pure to mixed star transition.

Next we present two estimates of $\frac{\dot{M}}{M}$.

First estimate.-The first estimate uses the average value $\frac{\Delta M}{M T} \approx \Gamma_{n n^{\prime}} \frac{\Delta M}{M}$ during the complete transition to a mixed star, where $T$ and $\Delta M$ are the total transition time and mass reduction. The $\frac{\Delta M}{M}=0.09-0.14$ obtained in the previous section then yields

$$
\frac{\dot{M}}{M} \approx 0.12 \Gamma_{n n^{\prime}}
$$

Second estimate.-Here we use the results of the numerical solutions of the neutron stars (pure and totally mixed) presented in Sec. IV. We apply Eq. (2) to the very beginning of the transition process. We find

$$
\left|\frac{\dot{M}}{M}\right| \geq\left(e^{\phi(R)}-e^{\phi(0)}\right) \Gamma_{n n^{\prime}}=(0.15 \div 0.19) \Gamma_{n n^{\prime}} .
$$

It is reassuring that the estimates by the two methods agree up to a factor of 1.5. It also makes sense that at the very beginning the process is faster than that derived from method 1 . This is because the rate of mass change vanishes towards the end of a transition to a mixed star with an equal

TABLE I. Limits on $\epsilon^{\prime}$ from several pulsar data using Eqs. (5) and (11). We assume that the $n-n^{\prime}$ conversion has not been completed in these pulsars.

\begin{tabular}{lccc}
\hline \hline Pulsar name & Age in yrs & Upper limit on $|\dot{M} / M|$ in $\mathrm{yr}^{-1}$ & $\begin{array}{c}\text { Upper limit on } \epsilon^{\prime} \text { in eV } \\
\text { [using Eq. (10)] }\end{array}$ \\
\hline PSR $1913+16$ & $1.1 \times 10^{8}$ & $6.8 \times 10^{-12}$ & $2.8 \times 10^{-13}$ \\
PSR $J 1141-6545$ & $2 \times 10^{6}$ & $1.6 \times 10^{-12}$ & $1.4 \times 10^{-13}$ \\
PSR J0437-4715 & $1.6 \times 10^{9}$ & $1.6 \times 10^{-11}$ & $4.4 \times 10^{-13}$ \\
PSR J1952+2630 & $7.7 \times 10^{7}$ & $7 \times 10^{-12}$ & $2.9 \times 10^{-13}$ \\
\hline \hline
\end{tabular}


number of $n \rightarrow n^{\prime}$ and $n^{\prime} \rightarrow n$ transitions, and the total mass of the system with given $M_{B}$ reaches a minimum.

Thus, we adopt

$$
\left|\frac{\dot{M}}{M}\right| \geq 0.14 \Gamma_{n n^{\prime}}
$$

as a representative value during the entire transition episode.

In Table 1 we summarize the various upper limits on $\epsilon^{\prime}$ using Eqs. (5) and (11).

\section{COMMENT ON NEUTRON DECAY ANOMALY}

Taking into account the various uncertainties, the upper bound on $\epsilon^{\prime}$ that we find is $\epsilon^{\prime}<4.10^{-13} \mathrm{eV}$. In principle, laboratory experiments using neutron beams and/or bottles could be sensitive to even smaller $\epsilon^{\prime}$ values. Indeed, the relatively long free propagation or residence time in such devices has by now reached times of order $t \sim 1 \mathrm{sec}$, and

$$
t . \epsilon^{\prime} \sim 0.1
$$

is achieved even for $\epsilon^{\prime} \sim 10^{-16} \mathrm{eV}$. However, our limit still excludes a large range of the parameter space explaining the $\sim 1 \%$ effect of the neutron lifetime anomaly via $n n^{\prime}$ mixing. The point is that the last expression assumes that the short time limit applies which requires that $\Delta(E)=\delta(m)+\mu \cdot B \leq 10^{-15} \mathrm{eV}$. However, the lifetime anomaly was observed for a range of $B$ fields, the largest of which definitely violates the last condition.

\section{CONCLUDING REMARKS}

In this paper, we show that astrophysical data pertinent to precision pulsar timing strongly constrain a putative $n \rightarrow n^{\prime}$ transition. This allows us to restrict the mixing between the neutron and mirror neutron $\left(n \rightarrow n^{\prime}\right)$, which is possible in an almost exact mirror symmetric dark sector.

We find that the key parameter $\epsilon^{\prime}$ responsible for the $n \rightarrow n^{\prime}$ transition is restricted to below $(3 \pm 1.5) 10^{-13} \mathrm{eV}$. It is interesting that the four binary pulsars considered here yield quite similar limits on $\epsilon^{\prime}$. We emphasize that our limits do not exclude the parameter space which can be responsible for a 5.2 sigma anomaly reported in [29] (corresponding to oscillation time $10 \mathrm{~s}$ ).

An interesting contrast between the current pulsar timing limits and the limits that can be obtained from laboratory searches is that our limit is valid for $n-n^{\prime}$ mass differences $\delta m^{\prime}$ as large as $20 \mathrm{MeV}$, whereas the latter limits are valid only for much smaller splittings such as those caused by the local magnetic field difference between the visible and the mirror world.

In passing we note that larger values of $\delta m^{\prime}$ would be in line with the comment made by two of us in [5]. We pointed out that the mirror symmetry breaking at some high scale, which is required for consistency with big bang nucleosynthesis with temperatures $T^{\prime}=(0.2-0.4) T$ tending to a "trickle down" effect via loop diagrams, barring some finetuning, generates $\delta m^{\prime}$ values much bigger than what is usually assumed.

\section{ACKNOWLEDGMENTS}

We thank Mark Alford, Jim Buckley, and Richard Mushotsky for extensive discussions, and S. N. would like to thank Anca Turneau and David Milstead for the invitation to the 2018 ESS neutron meeting. In particular, we thank an anonymous referee for very pertinent comments that helped us to substantially improve the paper. The work of R. N. M. is supported by the US National Science Foundation under Grant No. PHY1620074. The work of I. G. is supported by the Afeka Research Fund.
[1] T. D. Lee and C. N. Yang, Phys. Rev. 104, 254 (1956); K. Nishijima (private communication); Y. Kobzarev, L. Okun, and I. Ya Pomeranchuk, Yad. Fiz. 3, 1154 (1966); M. Pavsic, Int. J. Theor. Polit. 9, 229 (1974); S. I. Blinnikov and M. Y. Khlopov, Astron. Zh. 60, 632 (1983); E. W. Kolb, D. Seckel, and M. Turner, Nature (London) 314, 415 (1985); R. Foot, H. Lew, and R. Volkas, Phys. Lett. B 272, 67 (1991).

[2] R. Foot and R. Volkas, Phys. Rev. D 52, 6595 (1995).

[3] Z. Berezhiani and R. N. Mohapatra, Phys. Rev. D 52, 6607 (1995).

[4] Z. Berezhiani, A. Dolgov, and R. N. Mohapatra, Phys. Lett. B 375, 26 (1996).
[5] R. N. Mohapatra and S. Nussinov, Phys. Lett. B 776, 22 (2018).

[6] Z. Berezhiani, P. Ciarcelluti, D. Comelli, and F. L. Villante, Int. J. Mod. Phys. D 14, 107 (2005); Z. Berezhiani, D. Comelli, and F. L. Villante, Phys. Lett. B 503, 362 (2001); A. Y. Ignatiev and R. R. Volkas, Phys. Rev. D 68, 023518 (2003).

[7] For a review and references, see R. Foot, Int. J. Mod. Phys. A 29, 1430013 (2014); Z. Berezhiani, in From fields to strings, edited by M. Shifman et al. (World Scientific, Singapore, 2005), Vol. 3, pp. 2147-2195.

[8] Z. Berezhiani and L. Bento, Phys. Rev. Lett. 96, 081801 (2006); Z. Berezhiani, Eur. Phys. J. C 64, 421 (2009). 
[9] A. P. Serebrov et al., Phys. Lett. B 663, 181 (2008); I. Altarev et al., Phys. Rev. D 80, 032003 (2009); V. Bondar (nEDM at PSI Collaboration), arXiv:1607.07293; for a summary of all the bounds on $n-n^{\prime}$ oscillation time, see Z. Berezhiani, R. Biondi, P. Geltenbort, I. A. Krasnoshchekova, V. E. Varlamov, A. V. Vassiljev, and O. M. Zherebtsov, Eur. Phys. J. C 78, 717 (2018).

[10] L. Broussard and Y. Kamyshkov, Proceedings of BLV 2017; Z. Berezhiani, M. Frost, Y. Kamyshkov, B. Rybolt, and L. Varriano, Phys. Rev. D 96, 035039 (2017).

[11] V. A. Kuzmin, Pis'ma Zh. Eksp. Teor. Fiz. 12, 335 (1970); S. L. Glashow, NATO Sci. Ser. B 61, 687 (1980); R. N. Mohapatra and R. E. Marshak, Phys. Rev. Lett. 44, 1316 (1980); 44, 1643(E) (1980).

[12] For recent reviews, see R. N. Mohapatra, J. Phys. G 36, 104006 (2009); D. G. Phillips II et al., Phys. Rep. 612, 1 (2016).

[13] M. Baldo-Ceolin et al., Z. Phys. C 63, 409 (1994).

[14] J. Gustafson et al. (Super-Kamiokande Collaboration), Phys. Rev. D 91, 072009 (2015); B. Aharmim et al. (SNO Collaboration), Phys. Rev. D 96, 092005 (2017).

[15] I. Goldman and S. Nussinov, J. High Energy Phys. 08 (2010) 091.

[16] M. Mannarelli, Z. Berezhiani, R. Biondi, and F. Tonnelli, Proceedings of the Nordita ESS Workshop (2018).

[17] Z. Berezhiani, Eur. Phys. J. C 79, 484 (2019).
[18] A. W. Steiner, J. M. Lattimer, and E. F. Brown, Astrophys. J. 722, 33 (2010).

[19] R. Cowsik and S. Nussinov, Phys. Lett. 101B, 237 (1981).

[20] C. B. Dover, A. Gal, and J. M. Richard, Phys. Rev. D 27, 1090 (1983).

[21] J. H. Jeans, Mon. Not. R. Astron. Soc. 85, 2 (1924).

[22] N. D. R. Bhat, M. Bailes, and J. P. W. Verbiest, Phys. Rev. D 77, 124017 (2008).

[23] J. P. W. Verbiest, M. Bailes, W. van Straten, G. B. Hobbs, R. T. Edwards, R. N. Manchester, N. D. R. Bhat, J. M. Sarkissian, B. A. Jacoby, and S. R. Kulkarni, Astrophys. J. 679, 675 (2008).

[24] P. Lazarus et al., Mon. Not. R. Astron. Soc. 437, 1485 (2014).

[25] M. Linares, arXiv:1910.09572.

[26] H. H. Huang and W. Becker, Astron. Astrophys. 463, L5 (2007).

[27] I. Goldman, R. N. Mohapatra, S. Nussinov, D. Rosenbaum, and V. Teplitz, Phys. Lett. B 725, 200 (2013).

[28] See, for example, C. Kouvaris and N. G. Nielsen, Phys. Rev. D 92, 063526 (2015); P. Ciarcelluti and F. Sandin, Phys. Lett. B 695, 19 (2011); J. Ellis, G. Hütsi, K. Kannike, L. Marzola, M. Raidal, and V. Vaskonen, Phys. Rev. D 97, 123007 (2018).

[29] Z. Berezhiani and F. Nesti, Eur. Phys. J. C 72, 1974 (2012). 